\title{
ARTICLE
}

Received 10 Jun 2015 | Accepted 9 Nov 2015 | Published 22 Dec $2015 \quad$ DOl: 10.1038/ncomms10150

OPEN

\section{Talc-dominated seafloor deposits reveal a new class of hydrothermal system}

Matthew R.S. Hodgkinson ${ }^{1,2}$, Alexander P. Webber ${ }^{1}$, Stephen Roberts ${ }^{2}$, Rachel A. Mills ${ }^{2}$, Douglas P. Connelly ${ }^{1}$ \& Bramley J. Murton ${ }^{1}$

The Von Damm Vent Field (VDVF) is located on the flanks of the Mid-Cayman Spreading Centre, $13 \mathrm{~km}$ west of the axial rift, within a gabbro and peridotite basement. Unlike any other active vent field, hydrothermal precipitates at the VDVF comprise $85-90 \%$ by volume of the magnesium silicate mineral, talc. Hydrothermal fluids vent from a 3-m high, 1-m diameter chimney and other orifices at up to $215^{\circ} \mathrm{C}$ with low metal concentrations, intermediate $\mathrm{pH}$ (5.8) and high concentrations $\left(667 \mathrm{mmol} \mathrm{kg}^{-1}\right.$ ) of chloride relative to seawater. Here we show that the VDVF vent fluid is generated by interaction of seawater with a mafic and ultramafic basement which precipitates talc on mixing with seawater. The heat flux at the VDVF is measured at $487 \pm 101 \mathrm{MW}$, comparable to the most powerful magma-driven hydrothermal systems known, and may represent a significant mode of off-axis oceanic crustal cooling not previously recognized or accounted for in global models.

\footnotetext{
${ }^{1}$ National Oceanography Centre, Waterfront Campus, Southampton SO14 3ZH, UK. ${ }^{2}$ Ocean and Earth Science, National Oceanography Centre Southampton, University of Southampton, Southampton SO14 3ZH, UK. Correspondence and requests for materials should be addressed to M.R.S.H. (email: matthew.hodgkinson@noc.soton.ac.uk).
} 
$\mathrm{H}$ ydrothermal activity at mid-ocean ridges is dominated by basalt-hosted, high-temperature, metal-rich vent systems driven by magmatic activity ${ }^{1}$. However, the discovery of ultramafic-hosted hydrothermal vent fields (for example, the high-temperature Rainbow and the low-temperature Lost City Vent Fields) demonstrates the diversity of hydrothermal activity associated with medium-slow spreading ridges ${ }^{2,3}$. Tectonic exposure of upper mantle and lower crustal rocks gives rise to a more heterogeneous basement than at intermediate-fast spreading ridges, and detachment faulting provides pathways to enhance hydrothermal circulation ${ }^{4,5}$.

A new class of hydrothermal system, the Von Damm Vent Field (VDVF), was discovered in the Caribbean during cruise JC044 of the RRS James Cook in April 2010, which hosts a community of hydrothermal vent fauna similar to those at the Mid-Atlantic Ridge ${ }^{6}$. The VDVF hydrothermal plume is rich in methane, has a significant Eh anomaly (indicative of reduced fluids) and is free of metallic particulates ${ }^{6}$. Here we describe how the unusual mineralogy of the VDVF results from mixing between moderate-temperature vent fluid and cold seawater. The processes leading to the formation of the VDVF may be widespread throughout medium to ultraslow mid-ocean spreading ridges medium-ultraslow spreading mid-ocean ridges and could play a significant role in the cooling and chemical exchange between oceanic crust and seawater.

\section{Results}

Geological setting. The VDVF is located on the western flanks of the ultraslow spreading Mid-Cayman Spreading Centre, the deepest spreading centre on Earth (Fig. 1a). It is situated at a depth of $2,280 \mathrm{mbsl}, 13 \mathrm{~km}$ to the west of the rift axis ${ }^{6,7}$ on the upper slopes of Mt Dent, an oceanic core complex (OCC) formed by detachment faulting (Fig. 1b). At this location, the basement age is estimated to be between 1 and $2 \mathrm{Ma}$ (based on distance from spreading centre and spreading rate) and comprises meta-gabbro, dolerite dykes and serpentinized peridotites that are partially covered by calcareous pelagic sediment ${ }^{7}$. The hydrothermally active area of the VDVF comprises three overlapping conical-shaped talc mounds, up to $75 \mathrm{~m}$ high and $150 \mathrm{~m}$ in diameter, aligned north-northwest-south-southeast (NNW-SSE) (Fig. 1c). The summit of the largest mound (Main

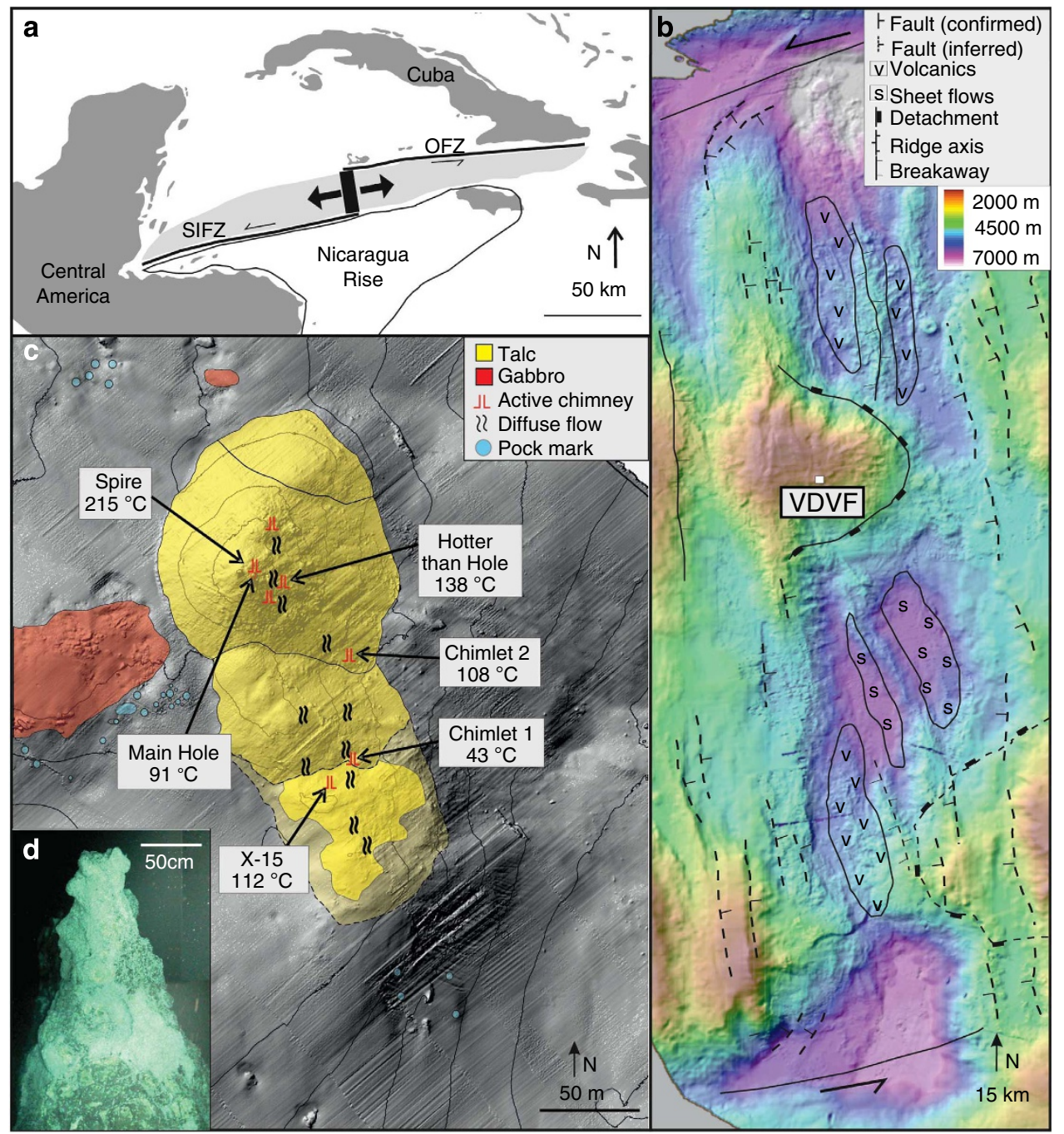

Figure 1 | Location and bathymetry of the VDVF. (a) map of the Caribbean showing the location of the Mid-Cayman Rise and the black rectangle represents the area of $\mathbf{b}$; the lightly shaded area is the area occupied of the Cayman Trough; OFZ, Oriente Fracture Zone; SIFZ, Swan Island Fracture Zone. (b) Bathymetry and interpretative geology of the Mid-Cayman Rise showing regional tectonic structures. (c) Bathymetry of the active VDVF showing the location of hydrothermal activity across the vent field. Contours are at 20-m intervals. (d) Photomosaic of The Spire at the top of the main VDVF cone obtained from high-definition video. 
Cone), which is the northern mound at the active site, hosts a 3-m tall, 1-m diameter chimney (The Spire, Fig. 1d). Hydrothermal fluid venting $\left(215^{\circ} \mathrm{C}\right)$ from The Spire has low concentrations of particles and a $\mathrm{pH}$ of 5.8 (determined at standard temperature and pressure (STP)). A 1-m diameter orifice (Main Hole) located at the base of The Spire vents fluids of up to $91^{\circ} \mathrm{C}$. Elsewhere across the VDVF, smaller orifices vent fluids of up to $138^{\circ} \mathrm{C}$ (for example, Hotter than Hole, Chimlets 1 and Chimlets 2-Fig. 1c). Talc rubble at the base of the Main Cone onlaps the surrounding calcareous sediment-a relationship reversed at the base of the southern mound, indicating an increase in the age of the mounds towards the south (Fig. 1c). A further series of hydrothermally inactive, conical-shaped talc mounds is located $700 \mathrm{~m}$ to the south and east of the three active mounds. These include a 90-m high, sediment-covered cone (Mystic Mountain), which has twice the volume of the Main Cone. Samples from these extinct mounds are similar in composition, mineralogy and texture to the active mounds. With an estimated sedimentation rate of between 2 and $5 \mathrm{~cm} \mathrm{ka}^{-1}$ (ref. 8), a thickness of $1 \mathrm{~m}$ or more of pelagic sediment covering most of Mystic Mountain indicates that its construction by hydrothermal activity ceased at least 20,000 years ago.

Petrology. Compared with hydrothermally active seafloor deposits elsewhere, the mounds and chimneys of the VDVF are highly unusual, constituting $85-90 \%$ talc by volume with up to $10 \%$ microcrystalline silica and $5 \%$ disseminated sulphides (Supplementary Fig. 1). In hand-specimen, the hydrothermally active chimneys show millimetric layers of laminated and botryoidal talc (Fig. 2a). These layers are parallel and have an internal colloform structure (Fig. 2a). Under scanning electron microscope (SEM), broken surfaces reveal dendritic networks and botryoidal masses of talc, indicative of growth into open void spaces (Fig. 2b,c). Concentric bands of sulphide up to $50-\mu \mathrm{m}$ thick are present as internal growth bands within the talc masses (Fig. 2d) and microcrystalline silica infills pore spaces (Fig. 2e). Talus forming the flanks of the mounds has a similar mineralogy to the venting chimneys, except that the botryoidal texture and open pore spaces are largely replaced by massive fine-grained talc, and the associated sulphides are mostly oxidized. Contrary to initial reports ${ }^{6}$, talc is the dominant mineralogy at the VDVF; only two of 50 samples recovered contain trace amounts of anhydrite and gypsum. Together with its botryoidal form, zoning, and layering. The talc deposits indicate precipitation from a hydrothermal fluid. The petrology of the hydrothermally active chimneys indicates a paragenetic sequence comprising initial talc growth into open space with co-precipitation of minor sulphide, followed by infilling of the pore space by later-stage microcrystalline silica. Loss of sulphides and a reduction in base-metal content in the flank talus indicate dissolution following exposure to cold and oxygenated seawater.

Mineralogy. Bulk X-ray diffraction analyses (Supplementary Fig. 1) and microscopy of the VDVF chimney and mound material confirm the dominant presence of talc, with microcrystalline silica and sulphide constituting up to $15 \%$. Chalcopyrite is the dominant sulphide (70\%), indicative of episodically higher vent temperatures ${ }^{9}$, with the remainder being $20 \%$ pyrite, $5 \%$ sphalerite and $5 \%$ galena. The presence of sulphides at the VDVF, and a lack of sulphate in the end-member fluids, confirms a reducing environment for the precipitation of the chimney and mound talc deposits ${ }^{10}$.

Other studies of talc, recovered from seafloor environments elsewhere, have revealed significant amounts of intracrystalline

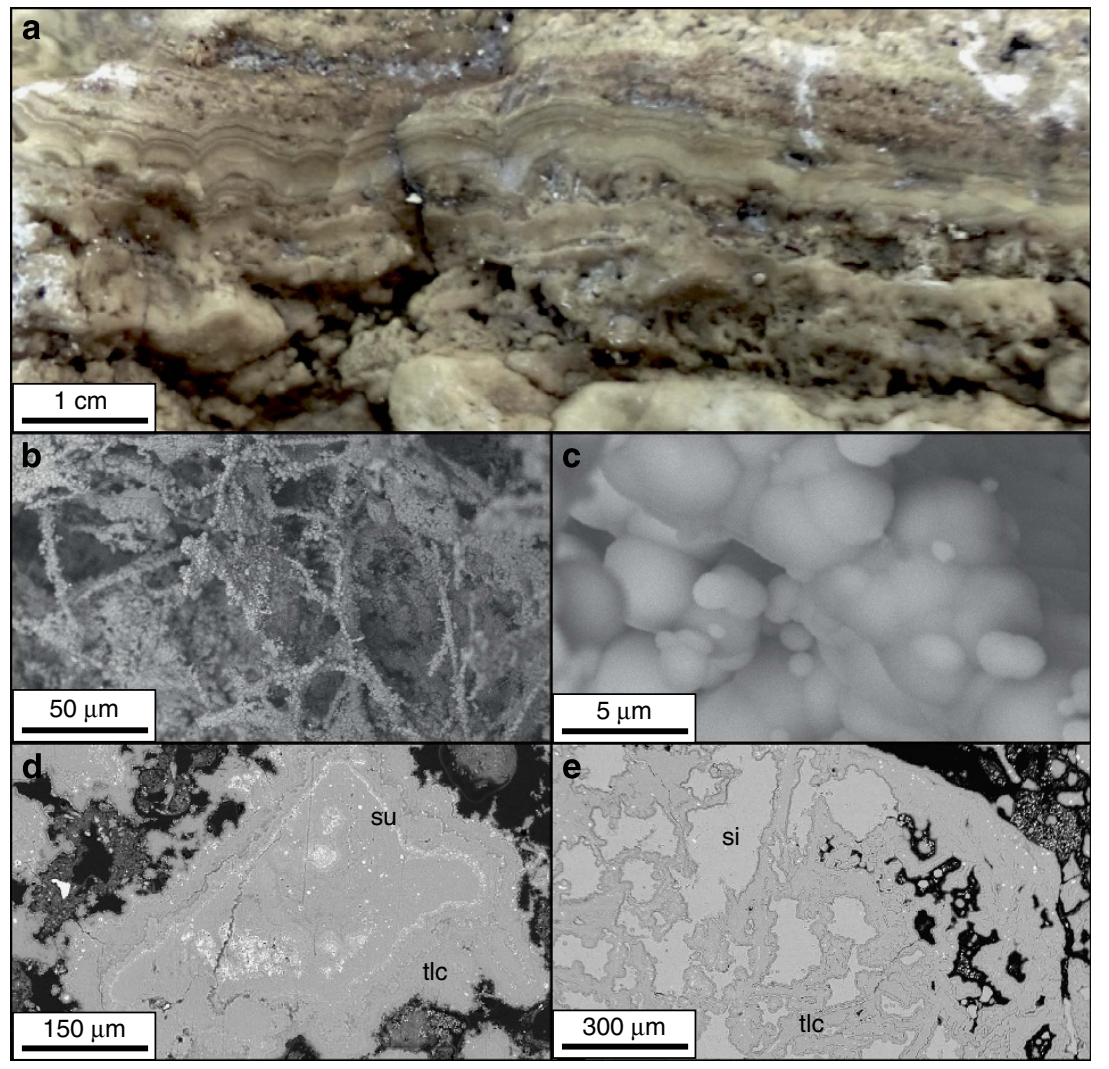

Figure 2 I Hand-specimen and SEM images of VDVF samples. (a) Collapsed chimney wall showing bands of massive talc. (b) and (c) SEM images of dendritic and botryoidal talc networks. (d) SEM image of a cross section through botryoidal talc with bands of sulphide. (e) SEM image showing microcrystalline silica precipitated into a talc framework with disseminated sulphides. Legend: $t \mathrm{c}=\mathrm{tal}$, su $=\mathrm{sulphide}$, si $=$ microcrystalline silica. 
layers of smectite clays reflecting the influence of sediment alteration and precipitation from $\mathrm{Mg}$-rich pore waters ${ }^{11}$. X-ray diffraction analyses of unorientated air-dried mounts of talc separates from the VDVF chimneys and talus deposits show peaks at $\sim 9.6 \AA$ (for the 001 plane) and $\sim 4.7 \AA$ (Supplementary Fig. 2) confirming talc $\left(\mathrm{Mg}_{3} \mathrm{Si}_{4} \mathrm{O}_{10}(\mathrm{OH})_{2}\right)$ as the dominant phase. Shifts in peaks of the 001 plane from 9.604-9.627 $\mathrm{A}$ for the air-dried mounts to $9.339-9.525 \AA$ for the glycolated mounts show the presence of up to $10 \%$ of a smectite clay that is interlayered within the crystalline talc structure. The presence of peaks at 1.529 and $1.532 \AA$ (for the 060 plane) indicate a tri-octahedral structure for the smectite clay interlayers (Supplementary Fig. 2). The dominance of talc (90\%), with up to $10 \%$ clay interlayers, contrasts with previously reported, sediment-hosted seafloor talc deposits ${ }^{11}$.

Deposit geochemistry. The high proportions of talc and microcrystalline silica are reflected in the whole-rock geochemistry (Table 1) by the high concentrations of $\mathrm{SiO}_{2}$ (52-66 wt.\%) and MgO, (25-34 wt.\%). Average base-metal concentrations in the hydrothermal chimneys are 1,463 p.p.m. $\mathrm{Cu}, 239$ p.p.m. $\mathrm{Zn}$ and 112 p.p.m. Pb. In contrast, concentrations in samples of mound talus are an order of magnitude lower with averages of 241 p.p.m. $\mathrm{Cu}, 48$ p.p.m. $\mathrm{Zn}$ and 15 p.p.m. $\mathrm{Pb}$, consistent with the observed oxidation and loss by dissolution of sulphides in the talus samples.

Rare earth element (REE) patterns for talc, separated from hydrothermally active chimneys and mound talus samples, have shallow U-shaped profiles dominated by a large and positive europium anomaly (Fig. 3). Enrichment in the light RREs (LREEs) is indicated by $\mathrm{La}_{(N)} / \mathrm{Sm}_{(N)}$ (where $N=$ chondritenormalized values) ratios ranging between 0.9 and 10.4 , with an average of 3.3 for chimneys and 3.1 for mound talus (Table 2). The REE profiles also have slight enrichment in heavy RRE (HREE) with average $\mathrm{Dy}_{(N)} / \mathrm{Yb}_{(N)}$ ratios of 0.8 for chimney material and 0.9 for the mound talus. The magnitude of the positive $\mathrm{Eu}$ anomaly, defined as $\mathrm{Eu} / \mathrm{Eu}^{*}$ (where $\mathrm{Eu} / \mathrm{Eu}^{*}=$ $\mathrm{Eu}_{(N)} / \sqrt{ }\left(\mathrm{Sm}_{(N)} \times \mathrm{Gd}_{(N)}\right)$, ranges between 6 and 228 , with an average of 99 for the chimneys and 58 for the mounds (Fig. 3 and Table 2). The VDVF chimney and talus talc have ${ }^{87} \mathrm{Sr} /{ }^{86} \mathrm{Sr}$ ratios of between 0.706313 and 0.709168 , respectively, which are similar to, but slightly less than, that of modern-day seawater $(0.7092)^{12}$. This contrasts with the surrounding meta-gabbros that have ${ }^{87} \mathrm{Sr} /{ }^{86} \mathrm{Sr}$ ratios of between 0.702902 and 0.703657 (Tables 2 and 3).

The U-shaped REE patterns and positive Eu anomalies in the chimney and mound material indicate talc precipitation from VDVF vent fluid that has some similarities to high-temperature 'black smoker' vent fluid REE chemistry ${ }^{13}$. Reducing conditions in the VDVF vent fluid, indicated by the presence of sulphides, enhance Eu mobility by the formation of divalent $\mathrm{Eu}$ chloride complexes, especially during the dissolution of plagioclase, resulting in large, positive $\mathrm{Eu}$ anomalies in fluids and precipitates ${ }^{14}$. Furthermore, the VDVF talc ${ }^{87} \mathrm{Sr} /{ }^{86} \mathrm{Sr}$ ratios indicate precipitation following mixing of the high-temperature VDVF vent fluid with a high proportion of seawater (of at least 10:1). Chlorinity in the end-member VDVF fluid of $667 \mathrm{mmol} \mathrm{kg}^{-1}$ is significantly elevated in comparison with seawater $\left(546 \mathrm{mmol} \mathrm{kg}^{-1}\right)$, enhancing the complexation of LREEs in relation to mid-RREs (MREEs) and HREEs in hydrothermal solutions at high temperatures and pressures, resulting in elevated $\mathrm{LREE}_{\text {talc }} / \mathrm{MREE}_{\text {talc }}$ ratios ${ }^{15}$. In contrast, moderate HREE enrichment is largely a crystallographic effect of the talc mineralogy, where HREEs substitute in the octahedral Mg site as a result of the ionic radii being of a more similar size compared with the LREEs ${ }^{16}$.
Other examples of seafloor talc deposits, reported from the St Paul and Conrad fracture zones, are inferred to have precipitated from the interaction of hydrothermal fluid with either seawater or a mafic protolith ${ }^{17}$. These talc samples have positive $\mathrm{Eu}$ anomalies and flat HREE profiles (Fig. 3), closely resembling those for the VDVF chimney and mound talus ${ }^{17}$. Seafloor talc deposits from elsewhere lack a positive Eu anomaly and have flat HREE profiles, consistent with formation as alteration products of an ultramafic protolith or sediment (Fig. 3) ${ }^{17,18}$. In contrast, the positive europium anomaly for the VDVF talc is consistent with primary precipitation from hydrothermal fluids. The radiogenic ${ }^{87} \mathrm{Sr} /{ }^{86} \mathrm{Sr}$ ratios for the talc further indicate that a significant component of seawater is mixed with the vent fluid during talc precipitation.

We conclude from the petrographic and geochemical evidence that the VDVF talc is a primary precipitate from a hydrothermal fluid mixed with seawater. To date, no other seafloor, talc-dominated, active hydrothermal vent field has been reported, making the discovery of the VDVF a new and unique class of hydrothermal system.

Vent fluid chemistry. To explore whether the VDVF is currently precipitating talc, we sampled and analysed the composition of the vent fluids escaping from three different chimneys with a maximum measured temperature range of $108-215^{\circ} \mathrm{C}$ (Table 3 ). It should be noted that the vent temperatures and fluid chemistry samples are decoupled, which precludes the possibility of extrapolating to an end-member temperature using fluid chemistry. Instead, we use the highest measured temperature of $226^{\circ} \mathrm{C}$ (ref. 19) and assume that this approaches the end-member temperature. We find that our vent fluid samples lie on a mixing line between seawater and zero Mg (Fig. 4). This is consistent with an end-member vent fluid from which $\mathrm{Mg}$ has been quantitatively removed in the subsurface ${ }^{20}$. When extrapolated to zero $\mathrm{Mg}$, the VDVF end-member fluid has a moderate $\mathrm{pH}$ of 5.8 (at STP; Table 3) and a dissolved $\mathrm{Si}$ concentration of $7.5 \mathrm{mmol} \mathrm{kg}^{-1}$ (ref. 21). End-member concentrations of $\mathrm{K}$ $\left(17.5 \mathrm{mmol} \mathrm{kg}^{-1}\right)$ and $\mathrm{Li}\left(241 \mu \mathrm{mol} \mathrm{kg}^{-1}\right)$ are much higher than those generated by phase separation of seawater alone (Fig. 4c and Table 3), indicating significant exchange with subsurface host rocks ${ }^{20}$. Strontium isotopes for the VDVF fluid samples also lie on a mixing line against $\mathrm{Mg} / \mathrm{Sr}$ with seawater indicating an end-member vent fluid ${ }^{87} \mathrm{Sr} /{ }^{86} \mathrm{Sr}$ of 0.702908 , close to the basement rock values of 0.702902-0.703657 (Fig. 4d and Tables 2 and 3). Base-metal concentrations in the fluid samples range from 6.6 to $604 \mu \mathrm{mol} \mathrm{kg}^{-1}$ for $\mathrm{Fe}, 4.6-14.2 \mu \mathrm{mol} \mathrm{kg}^{-1}$ for $\mathrm{Mn}$ and $0.4-460.0 \mu \mathrm{mol} \mathrm{kg}^{-1}$ for $\mathrm{Cu}$, but do not show conservative mixing with seawater and hence end-members cannot be derived (Table 3). Variation in base-metal concentrations and ratios between hydrothermal vents across the VDVF suggests subsurface processes of precipitation and/or zone refining within the talc mounds. Owing to highly variable concentrations, reliable end-member vent fluid $\mathrm{Fe}$ and $\mathrm{Mn}$ concentrations could not be determined. However, the range of concentrations of $\mathrm{Fe}$ and $\mathrm{Mn}$ are 10-1,000 times lower than those reported for the Rainbow 'black smoker' end-member vent fluid ${ }^{2}$. In contrast, a chlorinity of $667 \mathrm{mmol} \mathrm{kg}^{-1}$ for the end-member fluid is $22 \%$ higher than ambient seawater $\left(546 \mathrm{mmol} \mathrm{kg}^{-1}\right)$, indicating a process of brine concentration.

The low metal concentrations as well as the near-neutral $\mathrm{pH}$ of the fluids venting at VDVF are consistent with temperatures in the water-rock reaction zone, which are significantly cooler than the $\sim 500^{\circ} \mathrm{C}$ calculated for 'black smoker' vents ${ }^{22}$. It is also known that elevated hydrogen sulphide concentrations in 'black smoker' hydrothermal vents are related to magmatic input ${ }^{23}$. 
Table 1 | Whole-rock geochemistry.

\begin{tabular}{|c|c|c|c|c|c|c|c|c|c|c|c|c|}
\hline Type & Sample & $\begin{array}{c}\mathrm{SiO}_{2} \\
\text { (wt.\%) }\end{array}$ & $\begin{array}{c}\mathrm{TiO}_{2} \\
\text { (wt.\%) }\end{array}$ & $\begin{array}{r}\mathrm{Al}_{2} \mathbf{O}_{\mathbf{3}} \\
\text { (wt.\%) }\end{array}$ & $\begin{array}{l}\mathrm{Fe}_{2} \mathrm{O}_{3} \\
\text { (wt.\%) }\end{array}$ & $\begin{array}{c}\text { MgO } \\
\text { (wt.\%) }\end{array}$ & $\begin{array}{c}\mathrm{CaO} \\
\text { (wt.\%) }\end{array}$ & $\begin{array}{c}\mathbf{K}_{\mathbf{2}} \mathbf{O} \\
\text { (wt.\%) }\end{array}$ & $\begin{array}{c}\mathrm{Na}_{2} \mathrm{O} \\
\text { (wt.\%) }\end{array}$ & $\begin{array}{c}\mathbf{P}_{2} \mathbf{O}_{5} \\
\text { (wt.\%) }\end{array}$ & $\begin{array}{c}\text { LOI } \\
\text { (wt.\%) }\end{array}$ & $\begin{array}{c}\text { Sum } \\
\text { (wt.\%) }\end{array}$ \\
\hline Mound & 198-03 & 62.12 & 0.01 & 0.27 & 0.16 & 31.26 & 0.08 & 0.04 & 0.18 & 0.01 & 5.70 & 99.81 \\
\hline Mound & 198-04 & 56.54 & 0.01 & 2.75 & 1.15 & 30.08 & 0.20 & 0.09 & 1.27 & 0.00 & 7.66 & 99.75 \\
\hline Mound & 199-09 & 60.90 & 0.01 & 0.46 & 0.28 & 31.18 & 0.19 & 0.05 & 0.22 & 0.01 & 7.20 & 100.50 \\
\hline Mound & $199-10$ & 66.11 & 0.01 & 0.18 & 0.27 & 28.10 & 0.08 & 0.03 & 0.13 & 0.00 & 5.50 & 100.41 \\
\hline Mound & $200-21$ & 63.66 & 0.01 & 0.18 & 0.39 & 28.90 & 0.10 & 0.05 & 0.12 & 0.01 & 8.55 & 101.96 \\
\hline Mound & $201-34$ & 61.12 & 0.01 & 0.04 & 0.44 & 32.10 & 0.22 & 0.12 & 0.52 & 0.02 & 5.70 & 100.27 \\
\hline Mound & $201-36$ & 58.51 & 0.01 & 1.00 & 0.71 & 30.53 & 0.15 & 0.07 & 0.44 & 0.01 & 7.70 & 99.13 \\
\hline Chimney & $201-37$ & 53.51 & 0.01 & 0.24 & 0.57 & 30.71 & 0.06 & 0.07 & 0.24 & 0.02 & 9.63 & 95.04 \\
\hline Mound & $201-38$ & 58.39 & 0.01 & 0.31 & 0.29 & 31.77 & 0.37 & 0.09 & 0.38 & 0.01 & 9.10 & 100.70 \\
\hline Mound & 201-39 & 54.26 & 0.09 & 2.04 & 0.90 & 34.19 & 0.06 & 0.02 & 0.22 & 0.12 & 8.79 & 100.68 \\
\hline Mound & $201-40$ & 56.47 & 0.01 & 0.60 & 0.38 & 32.70 & 0.12 & 0.07 & 0.37 & 0.01 & 8.77 & 99.50 \\
\hline Chimney & $202-43$ & 57.99 & 0.01 & 0.19 & 0.33 & 31.81 & 0.18 & 0.10 & 0.43 & 0.01 & 8.30 & 99.33 \\
\hline Chimney & 199-107 & 58.31 & 0.01 & 0.65 & 0.64 & 30.92 & 0.15 & 0.07 & 0.40 & 0.01 & 8.78 & 99.94 \\
\hline Chimney & 199-110 & 57.05 & 0.01 & 0.19 & 0.19 & 30.73 & 0.21 & 0.08 & 0.41 & 0.02 & 11.02 & 99.91 \\
\hline Chimney & 44-1E & 58.12 & 0.01 & 0.44 & 1.13 & 27.81 & 0.12 & 0.09 & 0.59 & 0.01 & 11.50 & 99.80 \\
\hline Mound & $44-2 \mathrm{G}$ & 58.50 & 0.02 & 0.58 & 0.52 & 30.34 & 1.01 & 0.10 & 0.52 & 0.04 & 9.03 & 100.66 \\
\hline $\begin{array}{l}\text { As } \\
\text { (p.p.m.) }\end{array}$ & $\begin{array}{c}\text { Ba } \\
\text { (p.p.m.) }\end{array}$ & $\begin{array}{c}\text { Cd } \\
\text { (p.p.b.) }\end{array}$ & $\begin{array}{c}\text { Cs } \\
\text { (p.p.b.) }\end{array}$ & $\begin{array}{c}\mathrm{Cu} \\
\text { (p.p.m.) }\end{array}$ & $\begin{array}{c}M n \\
\text { (p.p.m.) }\end{array}$ & $\begin{array}{c}\mathbf{N b} \\
\text { (p.p.b.) }\end{array}$ & $\begin{array}{c}\mathbf{N i} \\
\text { (p.p.m.) }\end{array}$ & $\begin{array}{c}\mathbf{P b} \\
\text { (p.p.m.) }\end{array}$ & $\begin{array}{c}\mathbf{R b} \\
\text { (p.p.b.) }\end{array}$ & $\begin{array}{c}\text { Sb } \\
\text { (p.p.b.) }\end{array}$ & $\begin{array}{c}\text { Sr } \\
\text { (p.p.m.) }\end{array}$ & $\begin{array}{c}\text { Zn } \\
\text { (p.p.m.) }\end{array}$ \\
\hline 2.3 & 6.0 & 198 & 21 & 51.7 & 124.3 & 14 & 3.1 & 12.8 & 434 & 292 & 2.9 & 26.9 \\
\hline 1.0 & 7.3 & 26 & 28 & 44.8 & 107.6 & 16 & 1.2 & 7.4 & 710 & 44 & 16.8 & 14.4 \\
\hline 3.2 & 12.0 & 108 & 344 & 506.5 & 163.9 & 269 & 7.2 & 22.8 & 2,148 & 260 & 47.9 & 72.7 \\
\hline 1.0 & 0.3 & 14 & 40 & 82.5 & 151.2 & 22 & 2.6 & 1.2 & 636 & 44 & 8.9 & 12.3 \\
\hline 0.9 & 0.1 & 228 & 15 & 154.8 & 133.4 & 4 & 1.2 & 35.4 & 270 & 325 & 2.4 & 47.3 \\
\hline 8.1 & 0.8 & 2,028 & 107 & 970.2 & 463.9 & 23 & 4.7 & 116.6 & 1,216 & 3,767 & 11.0 & 234.7 \\
\hline 43.3 & 0.8 & 2,807 & 47 & 1,165.3 & 328.4 & 23 & 14.0 & 5.3 & 820 & 8,229 & 171.9 & 52.9 \\
\hline 75.3 & 0.8 & 2,791 & 51 & $1,663.4$ & 237.1 & 16 & 5.7 & 25.7 & 767 & 25,719 & 68.0 & 123.3 \\
\hline 5.8 & 0.3 & 848 & 35 & 471.1 & 175.2 & 4 & 1.6 & 20.2 & 495 & 4,703 & 7.2 & 77.9 \\
\hline 65.8 & 1.7 & 2,431 & 47 & $1,948.8$ & 277.6 & 4 & 6.5 & 31.6 & 839 & 20,425 & 154.3 & 144.2 \\
\hline 2.6 & 2.0 & 134 & 63 & $1,178.4$ & 205.0 & 84 & 2.7 & 23.1 & 819 & 442 & 9.8 & 43.3 \\
\hline 2.4 & 0.9 & 120 & 66 & $1,416.4$ & 240.5 & 109 & 2.6 & 16.8 & 1,154 & 257 & 14.1 & 33.1 \\
\hline 20.7 & 0.3 & 5,975 & 83 & $5,871.7$ & 212.9 & 12 & 9.7 & 658.9 & 873 & 25,042 & 8.4 & 1,272.7 \\
\hline 5.4 & 5.2 & 63 & 134 & 588.9 & 551.5 & 372 & 13.1 & 70.5 & 1,938 & 798 & 59.1 & 148.2 \\
\hline
\end{tabular}

ICP-AES, inductively couple plasma atomic emission spectroscopy; ICP-MS, inductively coupled plasma mass spectrometry; VDVF, Von Damm Vent Field; XRF, X-ray fluorescence.

Major and trace elements within hydrothermal active samples from the VDVF. Major elements were derived by XRF and trace elements derived by ICP-AES and ICP-MS.

Hence, we suggest the low $\mathrm{H}_{2} \mathrm{~S}$ concentrations at the VDVF are indicative of minimal magmatic contribution, consistent with the lower temperature of the end-member vent fluid and the ridge-flank setting of the vent field.

Increases in chlorinity for hydrothermal fluids interacting with an ultramafic basement have been suggested to occur in a number of ways: rock alteration, phase separation and brine and halite addition $^{2,24}$. Temperatures in excess of $360^{\circ} \mathrm{C}$ are required to phase-separate seawater at $200 \mathrm{bar}$ (that is, at the depth of the VDVF) and even higher for deep subsurface reactions ${ }^{25}$. This is considerably hotter than the maximum temperature recorded at the $\mathrm{VDVF}^{21}$ and would require substantial subsurface cooling by seawater circulating deep within the talc mounds for which there is no evidence. The dissolution of residual halite, or the mixing with residual brine formed during an earlier and higher-temperature period of hydrothermal circulation and phase separation deep in the crust, could increase the end-member chlorinity ${ }^{26,27}$; however, the VDVF and surrounding area lacks any mineralogical evidence for a 'black smoker'-like phase of venting. The process of serpentinization also has the potential to increase fluid chlorinity by the removal of water from the fluid $^{2,21}$. Using the equation for the serpentinization of pure forsterite to serpentine and brucite (equation (1)), we calculate that the increase in chlorinity for the VDVF end-member fluid 


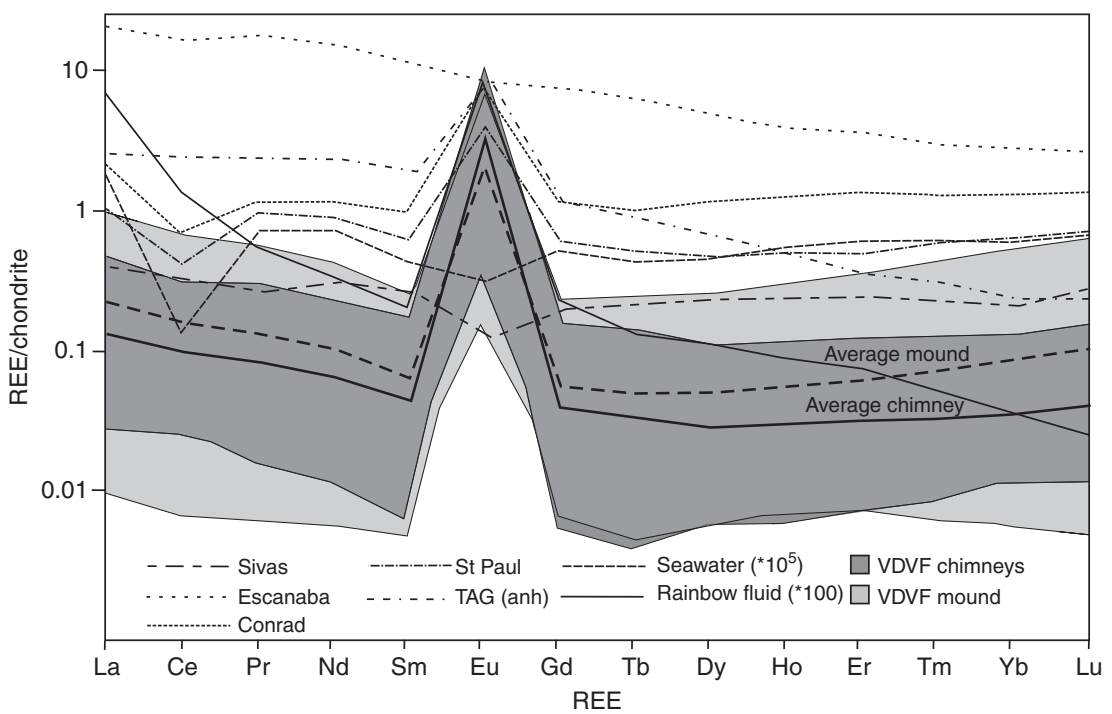

Figure 3 | REE plots. Chondrite-normalized REE patterns showing the range and average concentrations of the VDVF mound $(n=15)$ and chimney $(n=9)$ materials. The patterns are characterized by light and heavy REE enrichment and large positive Eu/Eu* anomalies. Also shown for comparison are samples from the St Paul Fracture Zone, Conrad Fracture Zone, Escanaba Trough, $(n=5)$, Sivas Basin $(n=2)$, TAG anhydrite $(n=24)$, Rainbow hydrothermal vent fluids $(n=2)$ and seawater $2,17,54-56$.

Table 2 | REE geochemistry.

\begin{tabular}{|c|c|c|c|c|c|c|c|c|c|c|c|c|c|c|c|c|}
\hline Type & $\begin{array}{l}\text { Sample } \\
\text { number }\end{array}$ & $\begin{array}{c}\text { La } \\
\text { (p.p.b.) }\end{array}$ & $\begin{array}{c}\mathrm{Ce} \\
\text { (p.p.b.) }\end{array}$ & $\begin{array}{c}\mathbf{P r} \\
\text { (p.p.b.) }\end{array}$ & $\begin{array}{c}\mathrm{Nd} \\
\text { (p.p.b.) }\end{array}$ & $\underset{\text { (p.p.b.) }}{\mathrm{Sm}}$ & $\begin{array}{c}\text { Eu } \\
\text { (p.p.b.) }\end{array}$ & $\begin{array}{c}\text { Gd } \\
\text { (p.p.b.) }\end{array}$ & $\begin{array}{c}\text { Tb } \\
\text { (p.p.b.) }\end{array}$ & $\begin{array}{c}\text { Dy } \\
\text { (p.p.b.) }\end{array}$ & $\begin{array}{c}\text { Ho } \\
\text { (p.p.b.) }\end{array}$ & $\begin{array}{c}\mathrm{Er} \\
\text { (p.p.b.) }\end{array}$ & $\underset{\text { (p.p.b.) }}{\operatorname{Em}}$ & $\begin{array}{c}Y b \\
\text { (p.p.b.) }\end{array}$ & $\begin{array}{c}\text { Lu } \\
\text { (p.p.b.) }\end{array}$ & ${ }^{87} \mathrm{Sr} /{ }^{86} \mathrm{Sr}$ \\
\hline Mound & 198-03 & 46.8 & 157.8 & 7.0 & 20.3 & 2.8 & 126.0 & 2.8 & 0.3 & 1.8 & 0.4 & 1.3 & 0.2 & 1.0 & 0.2 & 0.708685 \\
\hline Mound & 198-04 & 4.0 & 9.8 & 1.4 & 6.5 & 2.3 & 16.6 & 4.5 & 0.8 & 5.7 & 1.3 & 3.9 & 0.6 & 4.3 & 0.6 & 0.708937 \\
\hline Mound & 198-05 & 243.1 & 430.5 & 55.5 & 204.1 & 39.0 & 74.9 & 36.0 & 5.7 & 37.1 & 8.4 & 26.0 & 4.3 & 31.0 & 5.2 & N.D. \\
\hline Mound & 199-09 & 36.8 & 83.4 & 8.7 & 34.6 & 7.4 & 83.4 & 9.7 & 1.5 & 10.7 & 2.5 & 8.0 & 1.3 & 9.5 & 1.7 & N.D. \\
\hline Chimney & $199-10$ & 19.7 & 68.1 & 4.8 & 17.8 & 2.6 & 109.6 & 3.0 & 0.4 & 2.4 & 0.7 & 2.3 & 0.3 & 2.3 & 0.3 & N.D. \\
\hline Chimney & $199-107$ & 19.8 & 49.5 & 6.1 & 25.5 & 6.6 & 77.7 & 9.2 & 1.2 & 7.7 & 1.6 & 4.3 & 0.6 & 4.2 & 0.7 & N.D. \\
\hline Chimney & 199-108 & 7.3 & 17.6 & 2.0 & 9.0 & 2.4 & 20.9 & 3.4 & 0.5 & 3.6 & 0.8 & 2.6 & 0.4 & 2.8 & 0.5 & 0.709032 \\
\hline Chimney & $199-110$ & 12.1 & 28.2 & 3.6 & 15.0 & 3.5 & 58.2 & 5.0 & 0.8 & 5.3 & 1.3 & 4.1 & 0.7 & 6.2 & 1.2 & 0.709067 \\
\hline Mound & $200-23$ & 94.2 & 176.2 & 21.7 & 81.1 & 17.2 & 143.8 & 17.7 & 3.0 & 20.8 & 5.0 & 16.8 & 2.9 & 22.7 & 3.8 & 0.707526 \\
\hline Chimney & $200-24$ & 35.2 & 72.0 & 8.3 & 33.7 & 6.6 & 176.0 & 6.6 & 0.7 & 4.0 & 0.9 & 2.3 & 0.3 & 2.0 & 0.3 & 0.708375 \\
\hline Mound & 201-34 & 63.7 & 173.6 & 17.9 & 68.5 & 9.7 & 372.5 & 10.7 & 1.2 & 7.6 & 1.7 & 5.5 & 0.8 & 5.3 & 0.8 & N.D. \\
\hline Mound & $201-35$ & 7.7 & 26.7 & 3.3 & 16.4 & 5.3 & 21.2 & 6.9 & 1.1 & 6.4 & 1.3 & 3.9 & 0.6 & 5.0 & 0.8 & 0.708949 \\
\hline Mound & $201-36$ & 23.5 & 52.1 & 5.6 & 22.1 & 4.5 & 75.2 & 5.1 & 0.6 & 3.3 & 0.7 & 1.5 & 0.2 & 1.1 & 0.2 & 0.708770 \\
\hline Mound & $201-38$ & 11.4 & 28.3 & 3.4 & 15.9 & 5.0 & 77.7 & 8.6 & 1.2 & 7.8 & 1.7 & 4.5 & 0.6 & 3.9 & 0.7 & 0.709083 \\
\hline Mound & $201-39$ & 12.5 & 25.5 & 3.0 & 12.2 & 1.9 & 102.9 & 2.3 & 0.2 & 1.9 & 0.4 & 1.3 & 0.2 & 1.3 & 0.2 & N.D. \\
\hline Mound & $201-40 a$ & 5.9 & 11.0 & 1.7 & 7.2 & 1.7 & 94.8 & 3.3 & 0.5 & 4.0 & 1.1 & 3.7 & 0.5 & 3.6 & 0.5 & 0.709168 \\
\hline Mound & $201-40 b$ & 2.6 & 4.5 & 0.6 & 2.9 & 0.8 & 38.9 & 1.4 & 0.2 & 1.5 & 0.4 & 1.3 & 0.2 & 1.0 & 0.1 & N.D. \\
\hline Chimney & $202-43$ & 21.0 & 30.3 & 4.3 & 16.8 & 3.4 & 275.3 & 4.4 & 0.6 & 4.6 & 1.2 & 3.6 & 0.6 & 3.3 & 0.6 & 0.708824 \\
\hline Chimney & $202-47$ & 8.5 & 19.5 & 1.7 & 6.1 & 1.0 & 84.2 & 1.2 & 0.2 & 1.7 & 0.4 & 1.3 & 0.2 & 2.2 & 0.4 & 0.708308 \\
\hline Mound & $202-48$ & 3.7 & 11.0 & 1.4 & 6.7 & 2.0 & 9.6 & 3.3 & 0.5 & 3.8 & 0.9 & 2.9 & 0.5 & 3.2 & 0.6 & 0.706313 \\
\hline Chimney & $44-1 A$ & 114.9 & 193.9 & 28.8 & 109.3 & 26.4 & 617.4 & 32.2 & 5.3 & 27.8 & 6.7 & 20.7 & 3.2 & 21.6 & 4.0 & N.D. \\
\hline Chimney & $44-1 F$ & 48.7 & 85.6 & 12.3 & 47.4 & 8.2 & 260.0 & 9.8 & 1.7 & 9.7 & 2.2 & 7.3 & 1.4 & 9.1 & 1.4 & N.D. \\
\hline Mound & $44-2 C$ & 73.4 & 153.5 & 19.3 & 76.3 & 15.9 & 119.7 & 15.7 & 2.5 & 18.5 & 4.7 & 16.5 & 3.6 & 36.3 & 7.7 & N.D. \\
\hline Mound & $44-20$ & 164.0 & 179.3 & 39.8 & 158.3 & 31.9 & 404.1 & 47.7 & 9.2 & 65.2 & 17.1 & 57.7 & 11.2 & 88.7 & 16.3 & N.D. \\
\hline Host & $199-11$ & $1,149.7$ & $3,549.6$ & 535.8 & $2,545.0$ & 762.8 & 387.0 & 948.8 & 167.4 & $1,116.2$ & 238.1 & 710.7 & 111.6 & 773.9 & 119.1 & 0.702902 \\
\hline Host & 199-14 & $1,702.2$ & $6,215.1$ & $1,121.0$ & $6,194.4$ & $2,104.9$ & 826.2 & $2,748.4$ & 485.8 & $3,155.3$ & 660.1 & $1,893.2$ & 282.3 & $1,822.6$ & 265.7 & 0.703189 \\
\hline Host & $199-18$ & $6,570.5$ & $30,208.5$ & $2,562.0$ & $12,293.1$ & $3,551.1$ & $1,212.2$ & $4,294.8$ & 736.2 & $4,569.9$ & 948.2 & $2,621.5$ & 383.0 & $2,394.7$ & 345.8 & 0.703657 \\
\hline Host & 199-19 & $1,951.4$ & $7,873.6$ & 821.3 & $4,272.1$ & $1,495.6$ & 742.1 & $2,087.1$ & 365.4 & $2,358.3$ & 501.0 & $1,378.8$ & 199.7 & $1,262.0$ & 184.6 & 0.702997 \\
\hline
\end{tabular}

N.D., not determined; REE, rare earth element; VDVF, Von Damm Vent Field.

REE concentrations and ${ }^{87} \mathrm{Sr} /{ }^{86} \mathrm{Sr}$ in pure talc and host rock from the hydrothermally active VDVF. Samples were all recovered during cruises JC044 and JC082 (refs 57,58 ). Please refer to the Methods section for analytical precision and errors.

could result from serpentinization at a ratio of $944 \mathrm{~g}$ of forsterite to $1 \mathrm{~kg}$ seawater. The presence of any mineralogical $\mathrm{Cl}$ in serpentine makes this estimate a minimum.

$$
2 \mathrm{Mg}_{2} \mathrm{SiO}_{4}+3 \mathrm{H}_{2} \mathrm{O} \rightarrow \mathrm{Mg}_{3} \mathrm{Si}_{2} \mathrm{O}_{5}(\mathrm{OH})_{4}+\mathrm{Mg}(\mathrm{OH})_{2} \text {. }
$$

We have calculated a mass flux of end-member hydrothermal fluid at VDVF of $\sim 500 \mathrm{~kg} \mathrm{~s}^{-1}$ (see below) for which the increase in chlorinity requires brine expulsion following serpentinization of pure forsterite at a rate of at least $470 \mathrm{~kg} \mathrm{~s}^{-1}$. Alternatively, the entrainment of an early-formed brine or halite phase is consistent with a cooling hydrothermal system, while serpentinization is compatible with the tectonic setting of the VDVF on slowly exhumed lower-crust and upper-mantle rocks. Both processes are ultimately constrained by the availability of residual brine, halite or fresh peridotite.

Fluid pH. A measured $\mathrm{pH}$ of 5.8 (at STP) for the VDVF end-member fluid is significantly higher than the observed range ( $\mathrm{pH}$ 2.5-3.5) for sulphide-rich, high-temperature 'black smoker' vent fluids. It is also lower than the alkaline, low-temperature fluids vented at the ultramafic-hosted Lost City Vent Field ${ }^{3,20}$. 
Table 3 | Fluid data.

\begin{tabular}{|c|c|c|c|c|c|c|c|c|c|c|c|c|}
\hline \multicolumn{2}{|c|}{ Sample number } & \multirow{2}{*}{$\begin{array}{c}\text { 198-GT1 } \\
\text { The } \\
\text { Spire }\end{array}$} & \multirow{2}{*}{$\begin{array}{c}\text { 198-GT2 } \\
\text { The } \\
\text { Spire }\end{array}$} & \multirow{2}{*}{$\begin{array}{c}\text { 200-GT1 } \\
\text { Hotter than } \\
\text { Hole }\end{array}$} & \multirow{2}{*}{$\begin{array}{c}\text { 200-GT3 } \\
\text { Hotter } \\
\text { than Hole }\end{array}$} & \multirow{2}{*}{$\begin{array}{c}\text { 200-GT4 } \\
\text { Hotter than } \\
\text { Hole }\end{array}$} & \multirow{2}{*}{$\begin{array}{c}\text { 202-GT3 } \\
\text { Chimlet } \\
2\end{array}$} & \multirow{2}{*}{$\begin{array}{c}\text { 202-GT4 } \\
\text { Chimlet } \\
2 \\
\end{array}$} & \multicolumn{3}{|c|}{ End-members } & \multirow[t]{2}{*}{ Seawater } \\
\hline Site & & & & & & & & & VDVF & Rainbow & Lost City & \\
\hline Depth & $\mathrm{m}$ & 2,291 & 2,291 & 2,307 & 2,308 & 2,308 & 2,379 & 2,379 & $2,379-2,291$ & 2,300 & $700-800$ & \\
\hline $\begin{array}{l}\text { Max } \\
\text { temperature }\end{array}$ & ${ }^{\circ} \mathrm{C}$ & 215 & 215 & 138 & 138 & & 108 & 108 & 215 & 365 & & 4 \\
\hline $\mathrm{pH}$ & & 6 & 6.2 & 6.2 & 6. & 6.2 & 6.2 & 7 & 5.8 & 2.7 & 9 & 8.2 \\
\hline $\mathrm{Cl}$ & $\mathrm{mmol} \mathrm{kg}^{-1}$ & 643 & 592 & & & & & 574 & 667 & 745 & 541 & 546 \\
\hline $\mathrm{Mg}$ & $\mathrm{ol} \mathrm{kg}^{-1}$ & 14.7 & 31.4 & & 2 & 2 & 22.7 & 40 & 0 & 0 & $0-$ & 52.8 \\
\hline $\mathrm{Ca}$ & $\mathrm{mmol} \mathrm{kg}^{-1}$ & 15 & 13.4 & 12.9 & 13.1 & 13.2 & 13.4 & 11. & 16.4 & 66.6 & $26.6-27.4$ & 10.3 \\
\hline $\mathrm{Na}$ & $\mathrm{mmol} \mathrm{kg}^{-1}$ & 555 & 513 & 531 & 534 & 536 & 519 & 48 & 589 & 553 & 49.4 & 469 \\
\hline K & $\mathrm{mmol} \mathrm{kg}^{-1}$ & 15.5 & 13.1 & 14.1 & 14.1 & 13. & 13.6 & 11. & 17.5 & $20.2-20.4$ & 10.5 & 10.2 \\
\hline $\mathrm{Fe}$ & $\mu \mathrm{mol} \mathrm{kg}^{-1}$ & 18.9 & 6.6 & 138 & 604 & 392 & 144 & 16 & N.D. & $23,600-25,000$ & $<0.01$ & 0.001 \\
\hline $\mathrm{Sr}$ & $\mu \mathrm{mol} \mathrm{kg}-1$ & 92.8 & 91.0 & 90.9 & 90.6 & 90.1 & 88.0 & 86. & N.D. & 200 & N.D. & 91 \\
\hline $\mathrm{Mn}$ & $\mathrm{ol} \mathrm{kg}^{-1}$ & 8 & 4.6 & & 12 & 14.2 & 11 & 9. & $\mathrm{~N}$ & 2,200 & & 0 \\
\hline $\mathrm{Ba}$ & $\mathrm{kg}^{-1}$ & 5.55 & 2.61 & & & & & 2.52 & & 59 & $\mathrm{~N}$ & 0.07 \\
\hline $\mathrm{Li}$ & $\mathrm{kg}^{-1}$ & 175 & 110 & 1 & 140 & 145 & 137 & 69.6 & 241 & $327-345$ & $43-$ & 24.5 \\
\hline $\mathrm{Cu}$ & $\mu \mathrm{mol} \mathrm{kg}{ }^{-1}$ & 1.11 & 0.46 & 94.5 & 460 & 289 & 4.56 & 1.39 & N.D. & $121-162$ & N.D. & $<0.001$ \\
\hline $\mathrm{H}_{2} \mathrm{~S}$ & $\mathrm{mmol} \mathrm{kg}^{-1}$ & 0.927 & 0.898 & 0.332 & 0.439 & 0.967 & 0.635 & n.d. & N.D. & 1.2 & 0.06 & $<0.001$ \\
\hline $\mathrm{SO}_{4}$ & $\mathrm{mmol} \mathrm{kg}^{-1}$ & 7.85 & 15.1 & 12.5 & 11.7 & 11.4 & 10.6 & 20.1 & 0 & 0 & 0 & 28.2 \\
\hline${ }^{87} \mathrm{Sr} /$ & & N.D. & 0.706725 & 0.706174 & 0.705989 & 0.705958 & 0.705873 & 0.707801 & 0.702908 & N.D & N.D & 0.7092 \\
\hline $\mathrm{K} / \mathrm{Cl}$ & & 0.024 & 0.022 & 0.023 & 0.024 & 0.023 & 0.023 & 0.02 & 0.026 & 0.027 & 0.019 & 0.019 \\
\hline
\end{tabular}

N.D., not determined; VDVF, Von Damm Vent Field.

VDVF fluid dissolved elemental concentrations from three hydrothermal vents around the active VDVF, with the end-member vent fluid obtained by extrapolation to zero Mg concentration. End-member hydrothermal vent fluid data from the Lost City and Rainbow hydrothermal fields are included for comparison $2,3,38,59$
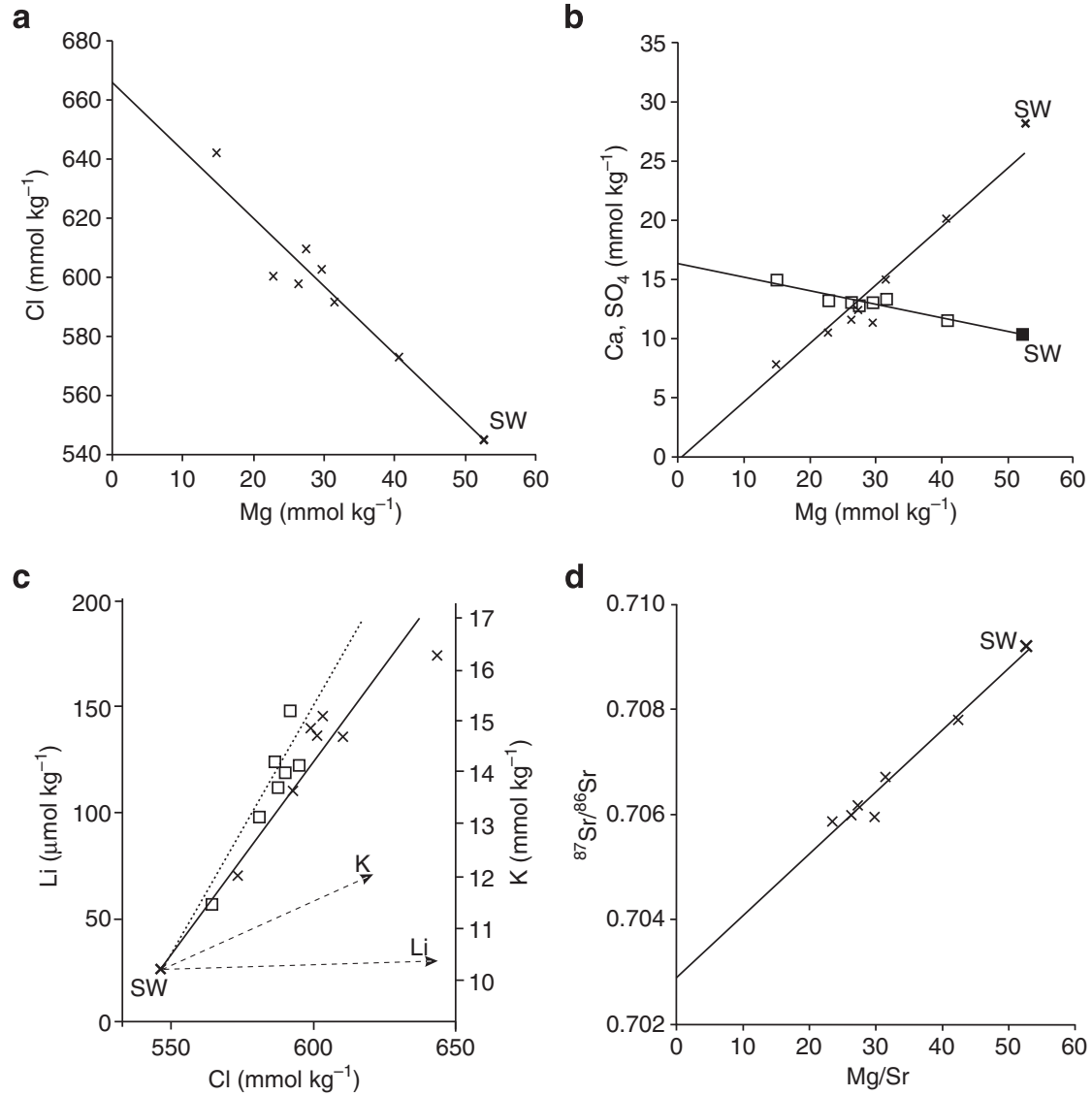

Figure 4 I VDVF fluid plots. (a,b) The top diagrams show $\mathrm{Cl}, \mathrm{SO}_{4}$ (crosses) and $\mathrm{Ca}$ (squares) with best fit lines extrapolated to zero $\mathrm{Mg}$ concentration. (c) Plots of $\mathrm{Cl}$ versus Li and K. The solid line and crosses indicate the linear trend of Li towards seawater (SW); the dashed line and squares indicate the linear trend of $\mathrm{K}$ towards SW. The arrows indicate the expected increase in $\mathrm{Li}, \mathrm{K}$ and $\mathrm{Cl}$ concentration resulting from phase separation of seawater alone. (d) Ratios of ${ }^{87} \mathrm{Sr} /{ }^{86} \mathrm{Sr}$ plotted against $\mathrm{Mg}$ concentration in fluid samples and extrapolated to zero $\mathrm{Mg} / \mathrm{Sr}$ to determine the VDVF end-member vent fluid ratio. 
Hydrothermal fluids with intermediate $\mathrm{pH}$ (5-6) can result from the interaction of seawater with mafic and/or ultramafic lithologies at temperatures $<300^{\circ} \mathrm{C}$ (refs 28,29). Under these conditions, $\mathrm{pH}$ is controlled by the balance between $\mathrm{Mg}$ removal from seawater and silicate hydrolysis (equation (2)). At low water/rock ratios $(<10)$, the rate of magnesium consumption is relatively low, and silicate hydrolysis acts as a $\mathrm{pH}$ buffer (equation (3)). At high water/rock ratios $(>50), \mathrm{H}^{+}$is produced at a rate greater than it is used up in hydrolysis reactions, resulting in lower $\mathrm{pH}^{28}$.

$$
\begin{gathered}
3 \mathrm{Mg}^{2+}+4 \mathrm{SiO}_{2(\mathrm{aq})}+4 \mathrm{H}_{2} \mathrm{O}=\mathrm{Mg}_{3} \mathrm{Si}_{4} \mathrm{O}_{10}(\mathrm{OH})_{2}+6 \mathrm{H}^{+} \\
2(\mathrm{Na}, \mathrm{Ca})(\mathrm{Si}, \mathrm{Al})_{4} \mathrm{O}_{8}+2 \mathrm{H}^{+}+\mathrm{K}^{+} \\
=\mathrm{KAl}_{2}\left(\mathrm{AlSi}_{3} \mathrm{O}_{10}\right)(\mathrm{OH})_{2}+\mathrm{Ca}^{2+}+\mathrm{SiO}_{2}+\mathrm{Na}^{+}
\end{gathered}
$$

With its intermediate $\mathrm{pH}, 215^{\circ} \mathrm{C}$ temperature and setting on lower-crustal and upper-mantle rocks, the fluids venting at the VDVF are consistent with moderate-temperature interaction within a gabbro/peridotite basement (equation (3)).

Talc precipitation. Thermodynamic modelling using the Geochemist's Workbench and SUPCRT92 (refs 30-32), under the ambient pressure and temperature conditions of the VDVF, predicts instantaneous precipitation of talc and silica as the primary phases on mixing the $215^{\circ} \mathrm{C}$ VDVF end-member vent fluid with cold seawater (Fig. 5a). Both phases remain supersaturated throughout the mixing regime until seawater makes up $\sim 90 \%$, and the fluid reaches $\sim 25^{\circ} \mathrm{C}$ (Fig. 5b). Below this temperature, talc remains undersaturated that, together with the kinetics of the reaction, may explain why talc was not visibly precipitating in the hydrothermal plume. Oscillatory zoning of talc and silica within active chimneys indicates a fluid composition at the VDVF that fluctuates around the intersection of the talc-silica saturation limits in the $\mathrm{H}_{2} \mathrm{O}-\mathrm{HCl}-\left(\mathrm{Al}_{2} \mathrm{O}_{3}\right)-\mathrm{MgO}-\mathrm{SiO}_{2}$ system. A fluid composition around the eutectic between talc and amorphous silica would be influenced by slight changes in $\mathrm{Mg}$ or Si activity, brought about either by precipitation of one or the other phase, or by fluctuating seawater proportions within the mound. Silica is saturated in the end-member fluid, therefore would precipitate when no seawater is available to provide $\mathrm{Mg}$ for talc precipitation. Such dynamic conditions, which are well documented at other sites ${ }^{33-35}$, could lead to alternating layers of mineral phases. Bands of chalcopyrite also indicate episodically higher temperatures. The prediction that talc precipitates directly as a result of mixing between the VDVF vent fluid and seawater is further supported by the range of ${ }^{87} \mathrm{Sr} /{ }^{86} \mathrm{Sr}$ ratios for the talc (0.706313-0.709168), which lie between the value of modern seawater (0.7092) and the VDVF end-member hydrothermal fluid (0.702908; Tables 2 and 3 and Fig. 4).

The dissolved silica concentrations in the VDVF end-member fluids are similar to those reported from 'black smoker' vents ${ }^{20}$, where talc is also theoretically stable on mixing with cold seawater. However, the large quantity of sulphides precipitated at these vent sites results in talc and other silicates only occurring as accessory minerals (for example, Middle Valley) ${ }^{36}$. At the VDVF, the low metal content of the fluid results in only accessory amounts of metal sulphides, allowing talc and silica to become the dominant phases. By comparison, the higher $\mathrm{pH}$ of the Lost City vent fluids results in calcium carbonate and brucite precipitation instead of talc ${ }^{3}$.

In summary, the geochemistry of the solid and fluid phases and thermodynamic modelling are consistent with the observed texture of the talc, its growth into pore spaces, the presence of actively venting talc chimneys and the precipitation of significant volumes of talc-forming large conical mounds on the seafloor.

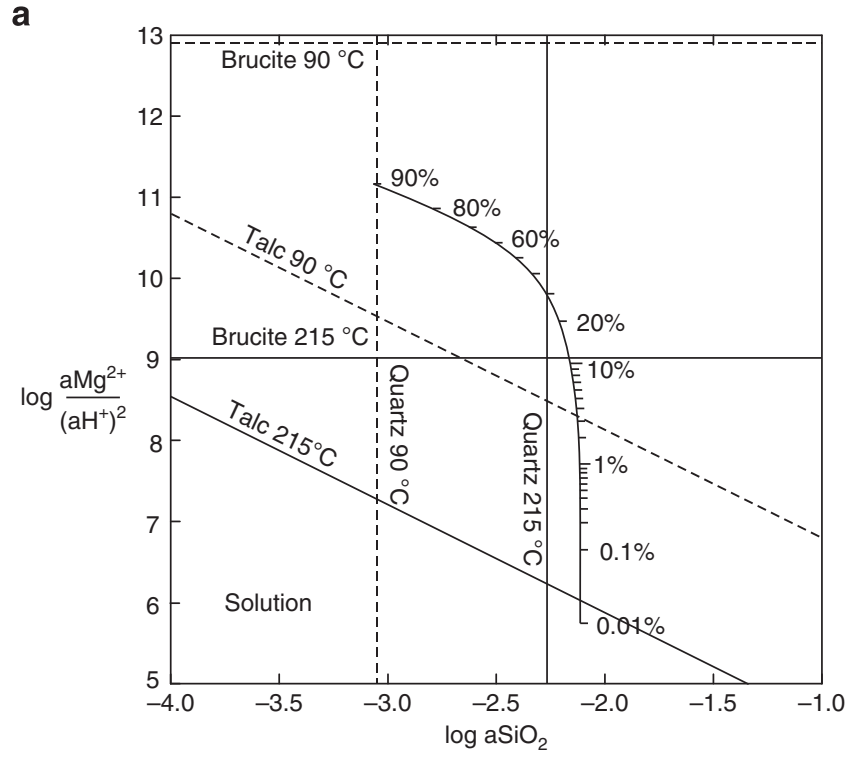

b

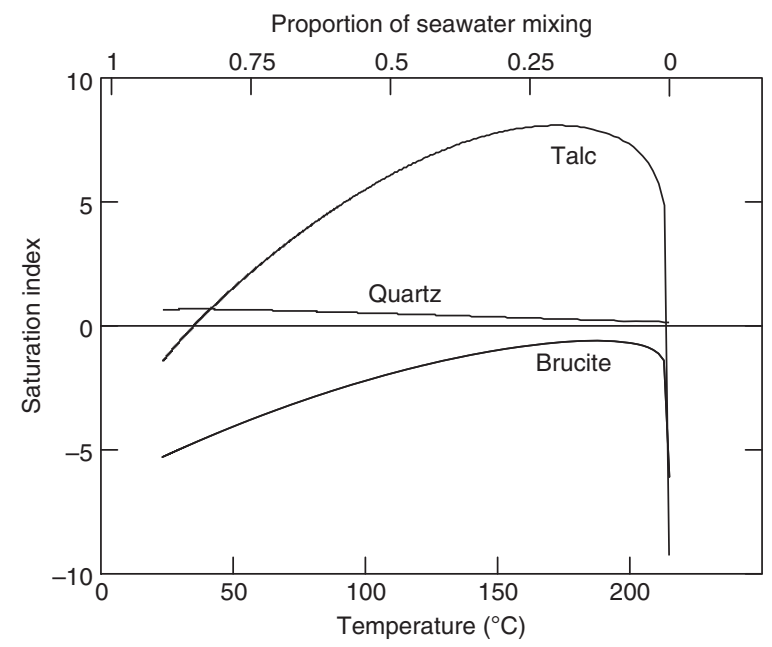

Figure 5 | Thermodynamic phase diagram. (a) Stability boundaries of talc, quartz and brucite at 215 and $90^{\circ} \mathrm{C}$, calculated in Geochemist's Workbench (GWB) at the ambient pressure conditions of the seafloor at the VDVF. The percentages indicate the proportion of seawater mixing with the endmember VDVF fluid. (b) Saturation indices for talc, quartz and brucite during modelled mixing of VDVF end-member fluid with seawater from 0.01 to $99 \%$. This indicates that talc becomes saturated on mixing endmember VDVF vent fluid with seawater, and remains stable throughout the mixing regime. Brucite, which is the dominant magnesium phase at the higher pH Lost City vent field, never reaches stability at the VDVF.

We suggest that similar conditions prevailed during the formation of the other, now hydrothermally extinct, talc mounds (for example, Mystic Mountain) located $\sim 700 \mathrm{~m}$ to the east of the currently active site. The current VDVF represents the latest stage in talc precipitation and hydrothermal circulation in the mafic and ultramafic basements beneath the Mt Dent OCC over tens of thousands of years.

\section{Discussion}

Hydrothermal venting on ocean crust more than $1 \mathrm{Ma}$ in age has only been observed previously as either low-temperature $\left(<10^{\circ} \mathrm{C}\right)$ diffuse flow of reduced fluids ${ }^{37}$ or as mediumtemperature $\left(91^{\circ} \mathrm{C}\right.$ ) venting of high $\mathrm{pH}$ fluids (for example, at 
the Lost City hydrothermal field ${ }^{3,38}$ ) forming brucite and carbonate chimneys. In contrast, the VDVF vents fluid in excess of $200^{\circ} \mathrm{C}$, from seafloor mounds and chimneys of predominantly talc, developed on a 1-2-Ma basement of lower-crust and upper-mantle rocks that were tectonically exhumed. This discovery, of an active hydrothermal system depositing predominantly talc as chimneys and forming large mounds on the seafloor, is unique.

While talc-rich deposits of postulated hydrothermal origin have been reported before ${ }^{11}$, these were mainly precipitated as accessory phases associated with massive sulphides, either where Si-rich low-pH hydrothermal fluids mixed with seawater, or as a result of conductive heating of $\mathrm{Mg}$-rich sedimentary pore waters. Only two seafloor talc deposits, from the St Paul's and Conrad fracture zones in the Atlantic Ocean ${ }^{17}$, appear directly comparable to the VDVF in terms of mineralogy, texture and geological setting.

At both sites, botryoidal talc, with similar REE patterns to the VDVF talc, was recovered from a tectonically exhumed gabbro-peridotite basement ${ }^{17}$. The presence of similar material to the active VDVF deposits at two different sites suggests that talc-dominated seafloor hydrothermal mineralization may be a widespread process at other slow spreading ridges, where tectonic uplift exposes lower-crustal and upper-mantle rocks to alteration by moderate-temperature hydrothermal circulation.

By measuring vertical velocities of vent fluid as it exits the seafloor, in situ fluid temperatures and the diameters of vent orifices at the VDVF, we have calculated a focused hydrothermal heat flux of $487 \pm 101 \mathrm{MW}$, produced from the venting of $\sim 500 \mathrm{~kg} \mathrm{~s}^{-1}$ of end-member fluid (Table 4). Despite being dissipated from crust of $1-2 \mathrm{Ma}$, this heat flux is comparable to that reported for focused heat flux dissipated by magma-driven 'black smoker' vent fields on zero-age ridge axes such as the Endeavour Ridge $(302 \mathrm{MW})$, the East Pacific Rise at $21^{\circ} \mathrm{N}$ $(200 \mathrm{MW})$ and the TAG vent field $(758 \mathrm{MW})$ on the Mid-Atlantic Ridge $^{39}$. When compared with other ridge-flank hydrothermal systems ${ }^{37}$, the heat flux at the VDVF is exceptionally high. It is also greater than the estimate for the Lost City hydrothermal field, which is thought to derive heat from the exothermic reaction of serpentinization ${ }^{40,41}$ in addition to residual crustal heat ${ }^{42}$.

While hydrothermal activity is effective at extracting heat from the magmatic ridge axis ${ }^{43,44}$, processes of residual heat extraction from ridge flanks remain unclear. The intermediate temperature, near-neutral $\mathrm{pH}$, low $\mathrm{H}_{2} \mathrm{~S}$ and low concentration of dissolved metals in the VDVF vent fluid are all features consistent with hydrothermal circulation driven by residual heat within the tectonically uplifted lower crust and upper mantle ${ }^{45}$. Despite a high heat flux, the neutrally buoyant VDVF hydrothermal plume contains very little particulate matter ${ }^{6}$ and, as a result, is difficult to detect using conventional optical sensors. Given the widespread occurrence of OCC's exhuming lower-crustal and upper-mantle rocks at ultraslow-medium spreading ridges, and the presence of similar talc deposits at the St Paul's and Conrad fracture zones ${ }^{17}$, we suggest that the processes leading to the formation of the VDVF could be widespread at slow-ultraslow spreading. In that case, the VDVF class of hydrothermal activity could contribute significantly to the cooling of the oceanic crust, but are not yet included in current estimates of global hydrothermal activity based on plume incidence ${ }^{46}$.

\section{Methods}

Sample collection. Sonar data, photographic and video imagery and samples of rocks, minerals, fluids and temperatures were acquired using remotely operated vehicles (Isis and HyBIS) during RRS James Cook cruises JC44 and JC82.

Vent fluids. High-temperature fluids were sampled using titanium gas-tight syringes at three different high-temperature chimneys (Table 3). Fluid exit temperatures were determined separately from the fluid sampling using a laboratory-calibrated, high-temperature thermal probe that was inserted deep into each vent orifice. Hence, the fluid samples and temperatures are decoupled. The highest measured value for each vent is reported in Table 3. Subsamples of vent fluids were taken for geochemical analyses, with $\mathrm{pH}$, halides, sulphate and $\mathrm{H}_{2} \mathrm{~S}$ being determined at STP $\left(25^{\circ} \mathrm{C}\right)$ immediately after recovery of the titanium syringes on the surface. Anions were determined using ion chromatography, and cations using inductively coupled plasma atomic emission spectroscopy and inductively coupled plasma mass spectrometry (ICP-MS) at NOCS. Data quality was assessed using the 'Mottl Vent Fluid Database' protocols ${ }^{47}$. This included analysis of all solid precipitates from within the sampling syringes following total dissolution and weighted volumetric addition of concentrations to the clear fluid samples. Hence, any precipitates after the fluid samples were taken were redissolved and accounted for in final concentrations. Quality control included screening for samples with excessive $\mathrm{Mg}$ on a regression of elements such as $\mathrm{Si}, \mathrm{Ca}$, $\mathrm{K}$ and $\mathrm{Li}$ to zero $\mathrm{Mg}$. Samples that were found to be contaminated with $\mathrm{Mg}$ concentrations more than those of seawater were subsequently excluded from the database. Our end-member concentration for dissolved silica is not as well constrained as that obtained in ref. 21 , and thus their concentration is cited and used in our thermodynamic modelling.

Petrography. Petrographic analyses were conducted using transmitted and reflected light microscopy on polished thin sections. SEM was conducted using two different systems: one using a low-resolution Hitachi TM1000 Desktop SEM operating at $15 \mathrm{kV}$, imaging $1-\mathrm{cm}^{2}$ blocks of untreated broken surfaces; the second was a high-resolution LEO 1450-VP SEM operating at $20 \mathrm{kV}$ and imaging carboncoated polished thin sections. Crystallographic analysis was made using X-ray diffraction with $\mathrm{Cu} \mathrm{K} \alpha$ radiation $2 \theta$ ranging between $2^{\circ}$ and $76^{\circ}$, at steps of $0.02^{\circ}$ and at rates of $0.02^{\circ}$ per s. Unorientated air-dried mounts and glycolated mounts were scanned in the ranges $0-20^{\circ}$ and $0-40^{\circ}$. Randomly orientated mounts were prepared and scanned in the range $57-62^{\circ}$ to identify the peak signifying the $(060)$ crystallographic plane.

Geochemistry. X-ray fluorescence (XRF) analysis was conducted on fused beads in a 10:1 ratio of sample powder to lithium tetraborate flux. Precision was determined from repeat analysis of standards UB-N, BRR-1 and OPY-1, and gives errors of $<10 \%$. Talc mineral separates were handpicked and washed in a weak acid solution and rinsed in de-ionized water. Following hydrofluoric and nitric acid digestion, the samples were analysed for trace element and REE analyses using ICP-MS. Standards included international reference materials: BIR-1, JB1a, JGB1, JB-3 and BHVO-2. Precision was determined from repeat analysis and gives errors in the range of $0.21-5.71 \%$. Whole-rock analysis was conducted using both ICP-MS and inductively couple plasma atomic emission spectroscopy, and calibration was made using synthetic multielement standards. ${ }^{87} \mathrm{Sr} /{ }^{86} \mathrm{Sr}$ ratios were

Table 4 | Heat flux calculation.

\begin{tabular}{|c|c|c|c|c|c|c|}
\hline Mound & Vent orifice & $\Delta T(\mathrm{~K})$ & Radius (m) & Vertical flow rate $\left(\mathrm{m} \mathrm{s}^{-1}\right)$ & Calculated flux & Units \\
\hline Main Cone & Main Hole & 87 & 0.5 & 1.5 & $401 \pm 80$ & MW \\
\hline Main Cone & Spire & 211 & 0.1 & 1.5 & $39 \pm 10$ & MW \\
\hline North spur & Chimlet 2 & 104 & 0.1 & 0.5 & $6 \pm 3$ & MW \\
\hline North spur & Chimlet 1 & 39 & 0.15 & 1 & $11 \pm 2$ & MW \\
\hline South spur & $X-15$ & 108 & 0.15 & 1 & $30 \pm 6$ & MW \\
\hline Total & & & & & $487 \pm 101$ & MW \\
\hline
\end{tabular}

bottom water $\left(4^{\circ} \mathrm{C}\right) \cdot \mathrm{C}$, specific thermal capacity of seawater at 200 bar and $215^{\circ} \mathrm{C}$ (that is, $\left.4,500 \mathrm{Jg}^{-1} \mathrm{~K}^{-1}\right)^{53}$. $\mathrm{M}$, mass flux $\left(\mathrm{kg} \mathrm{s} \mathrm{s}^{-1}\right)=A \times V \times \rho$, where $A$, area of vent orifice $\left(\mathrm{m}^{2}\right) ; V_{1}$ mean vertical flow rate $\left(\mathrm{m} \mathrm{s}^{-1}\right) ; \rho$, density of seawater at $215^{\circ} \mathrm{C}$ 
obtained using a thermal ionization mass spectrometer. Fluid and host rock samples were analysed by running through Sr-spec ${ }^{\mathrm{Tm}}$ resin to obtain $1 \mu \mathrm{g}$ of strontium. Samples of strontium $(200 \mu \mathrm{g})$ were obtained from pure talc by running through cation resin AG50-X8 200-400 and then through Sr-spec ${ }^{\mathrm{Tm}}$ resin. External reproducibility of analysis was checked by analysing standard NBS987 every 11 samples and is reported here with an average of 0.710250 $(1 \sigma=0.0000053)$, which is within an error of the accepted value of 0.710254 .

Thermodynamic modelling. Modelling was carried out using Geochemist's Workbench ${ }^{30}$ at pressure and temperature conditions appropriate for the depth of the VDVF. A thermodynamic database for the composition of the vent fluid and seawater, at 250 bar and $4-215^{\circ} \mathrm{C}$, with $\operatorname{logK}$ values calculated through this range of temperatures, was generated using DBCreate ${ }^{31}$, which in turn uses SUPCRT92 (ref. 32). Thermodynamic properties for aqueous species and phases were taken from the 2006 revision of the SUPCRT database ${ }^{32}$.

Heat flux calculation. Focused hydrothermal flow rates were calculated from high-definition video footage of the vertical velocity of particles entrained within venting hydrothermal fluid.

Individual particles entrained in the exiting vent fluid were filmed over a rise height of $\sim 1 \mathrm{~m}$ and their velocity calculated from frame to frame. The distance travelled by the particles was measured against two parallel laser beams separated by a constant distance of $10 \mathrm{~cm}$ and shone through the rising vent fluid. The fluid exit velocities at each vent orifice were determined by measuring multiple particles in the upflowing fluid across the entire dimensions of the vent orifices. Different particles entrained in the up-flow zone from each orifice displayed only slight variation in their velocities, indicating that the flow rates are relatively uniform for fluids exiting each vent orifice (Supplementary Table 1). The velocity of a number of particles was thus calculated over a period of several minutes and a mean velocity derived for vent fluid as it escaped the vent orifice. The calculated error in the velocities is derived from the variation in particle velocity. These data were then integrated for each orifice as per established methods ${ }^{48-52}$.

This method models the vent orifices as circles, and makes the assumptions that the fluid is emanating from the vent at a constant velocity across its diameter, the temperature is uniform across the vent orifice and the particle velocities recorded were representative of the flow velocity. Specific heat capacity of the venting fluids was calculated using specific densities and thermal capacities for seawater ${ }^{53}$ at the temperature and pressure conditions recorded at the VDVF.

\section{References}

1. Tivey, M. K. Generation of seafloor hydrothermal vent fluids and associated mineral deposits. Oceanography 20, 50-65 (2007).

2. Douville, E. et al. The Rainbow vent fluids $\left(36^{\circ} 14^{\prime} \mathrm{N}\right.$, MAR): the influence of ultramafic rocks and phase separation on trace metal content in Mid-Atlantic Ridge hydrothermal fluids. Chem. Geol. 184, 37-48 (2002).

3. Kelley, D. S. et al. An off-axis hydrothermal vent field near the Mid-Atlantic Ridge at $30^{\circ} \mathrm{N}$. Nature $412,145-149$ (2001).

4. Pedersen, R. B., Thorseth, I. H., Nygård, T. E., Lilley, M. D. \& Kelley, D. S. in Diversity Of Hydrothermal Systems On Slow Spreading Ocean Ridges Vol. 188 (eds Rona, P., Dewey, C. F., Dyment, J. \& Murton, B. J.) 67-89 (American Geophysical Union, 2010).

5. McCaig, A. M., Delacour, A., Fallick, A. E., Castelain, T. \& Frueh-Green, G. L. in Diversity of Hydrothermal Systems on Slow Spreading Ocean Ridges, Geophysical Monograph Series Vol. 188 (eds Rona, P., Dewey, C. F., Dyment, J. \& Murton, B. J.) 207-239 (American Geophysical Union, 2010).

6. Connelly, D. P. et al. Hydrothermal vent fields and chemosynthetic biota on the world's deepest seafloor spreading centre. Nat. Commun. 3, 620 (2012).

7. Hayman, N. W. et al. Oceanic core complex development at the ultraslow spreading Mid-Cayman Spreading Center. Geochem. Geophys. Geosyst. 12, Q0AG02 (2011)

8. Rosencrantz, E. \& Sclater, J. G. Depth and age in the Cayman trough. Earth Planet. Sci. Lett. 79, 133-144 (1986).

9. Seyfried, Jr W. E. \& Ding, K. The effect of redox on the relative solubilities of copper and iron in Cl-bearing aqueous fluids at elevated temperatures and pressures: an experimental study with application to subseafloor hydrothermal systems. Geochem. Cosmochim. Acta 57, 1905-1917 (1993).

10. Shanks, III W. C., Bischoff, J. L. \& Rosenbauer, R. J. Seawater sulfate reduction and sulfur isotope fractionation in basaltic systems: interaction of seawater with fayalite and magnetite at $200-350^{\circ} \mathrm{C}$. Geochem. Cosmochim. Acta 45, 1977-1995 (1981)

11. Dekov, V. M., Cuadros, J., Shanks, W. C. \& Koski, R. A. Deposition of talc-kerolite-smectite at seafloor hydrothermal vent fields: evidence from mineralogical, geochemical and oxygen isotope studies. Chem. Geol. 247, 171-194 (2008)

12. Palmer, M. R. \& Edmond, J. M. The strontium isotope budget of the modern ocean. Earth Planet. Sci. Lett. 92, 11-26 (1989).

13. Douville, E. et al. Yttrium and rare earth elements in fluids from various deepsea hydrothermal systems. Geochem. Cosmochim. Acta 63, 627-643 (1999).
14. Sverjensky, D. A. Europium redox equilibria in aqueous solution. Earth Planet. Sci. Lett. 67, 70-78 (1984).

15. Bao, S.-X., Zhou, H.-Y., Peng, X.-T., Ji, F.-W. \& Yao, H.-Q. Geochemistry of REE and yttrium in hydrothermal fluids from the Endeavour segment, Juan de Fuca Ridge. Geochem. J. 42, 359-370 (2008).

16. Shannon, R. Revised effective ionic radii and systematic studies of interatomic distances in halides and chalcogenides. Acta Crystallogr. 32, 751-767 (1976).

17. D'Orazio, M., Boschi, C. \& Brunelli, D. Talc-rich hydrothermal rocks from the St Paul and Conrad fracture zones in the Atlantic Ocean. Eur. J. Mineral. 16, 73-83 (2004).

18. Bach, W., Garrido, C. J., Paulick, H., Harvey, J. \& Rosner, M. Seawaterperidotite interactions: first insights from ODP Leg 209, MAR $15^{\circ}$ N. Geochem. Geophys. Geosyst. 5, Q09F26 (2004).

19. McDermott, J. M., Seewald, J. S., German, C. R. \& Sylva, S. P. Pathways for abiotic organic synthesis at submarine hydrothermal fields. Proc. Natl Acad. Sci. USA 112, 7668-7672 (2015).

20. Von Damm, K. L. in Physical, Chemical, Biological, and Geological Interactions within Hydrothermal Systems, Geophysical Monograph Series Vol. 91 (ed. Humphris, S.) 222-247 (American Geophysical Union, 1995).

21. McDermott, J. M. Geochemistry of Deep-Sea Hydrothermal Vent Fluids from the Mid-Cayman Rise, Caribbean Sea. Doctor of Philosophy thesis, Massachusetts Institute of Technology and Woods Hole Oceanographic Institute (2015).

22. Alt, J. C. in Seafloor Hydrothermal Systems: Physical, Chemical, Biological, and Geological Interactions, Geophysical Monograph Series Vol. 91 (eds Humphris, S., Zierenberg, R. A., Mullineaux, L. S. \& Thomson, R. E.) 85-114 (AGU, 1995).

23. Von Damm, K. et al. Evolution of East Pacific Rise hydrothermal vent fluids following a volcanic eruption. Nature 375, 47-50 (1995).

24. Von Damm, K. L. Seafloor hydrothermal activity: black smoker chemistry and chimneys. Annu. Rev. Earth Planet. Sci. 18, 173-204 (1990).

25. Bischoff, J. L. \& Rosenbauer, R. J. The critical point and two-phase boundary of seawater, $200-500^{\circ}$ C. Earth Planet. Sci. Lett. 68, 172-180 (1984).

26. Kelley, D. S., Robinson, P. T. \& Malpas, J. G. Processes of brine generation and circulation in the oceanic crust: fluid inclusion evidence from the Troodos Ophiolite, Cyprus. J. Geophys. Res. 97, 9307-9322 (1992).

27. Berndt, M. E. \& Seyfried, W. E. Calibration of $\mathrm{BrCl}$ fractionation during subcritical phase separation of seawater: possible halite at 9 to $10^{\circ} \mathrm{N}$ East Pacific Rise. Geochem. Cosmochim. Acta 61, 2849-2854 (1997).

28. Seyfried, Jr W. Experimental and theoretical constraints on hydrothermal alteration processes at mid-ocean ridges. Annu. Rev. Earth Planet. Sci. 15, 317 (1987).

29. Allen, D. E. \& Seyfried, Jr W. E. Compositional controls on vent fluids from ultramafic-hosted hydrothermal systems at mid-ocean ridges: an experimental study at $400^{\circ} \mathrm{C}, 500$ bars. Geochem. Cosmochim. Acta 67, 1531-1542 (2003).

30. Bethke, C. M. Geochemical reaction modelling: concepts and applications. Forensic Sci. Int. 198, 126-133 (1996).

31. Kong, X.-Z., Tutolo, B. M. \& Saar, M. O. DBCreate: a SUPCRT92-based program for producing EQ3/6, TOUGHREACT, and GWB thermodynamic databases at user-defined T and P. Comput. Geosci. 51, 415-417 (2013).

32. Johnson, J. W., Oelkers, E. H. \& Helgeson, H. C. SUPCRT92: a software package for calculating the standard molal thermodynamic properties of minerals, gases, aqueous species, and reactions from 1 to 5000 bar and 0 to 1000 C. Comput. Geosci. 18, 899-947 (1992).

33. Petersen, S., Herzig, P. M. \& Hannington, M. D. in Proceedings of the Ocean Drilling Program: Scientific Results, Vol. 158, (eds P. Herzig, S. Humphris, D. J. Miller, \& R. A. Zierenberg) 163-178 (Ocean Drilling Program, 1998).

34. Vanko, D. A., Bach, W., Roberts, S., Yeats, C. J. \& Scott, S. D. Fluid inclusion evidence for subsurface phase separation and variable fluid mixing regimes beneath the deep-sea PACMANUS hydrothermal field, Manus Basin back arc rift, Papua New Guinea. J. Geophys. Res. 109, B03201 (2004).

35. Webber, A. P., Roberts, S., Burgess, R. \& Boyce, A. J. Fluid mixing and thermal regimes beneath the PACMANUS hydrothermal field, Papua New Guinea: helium and oxygen isotope data. Earth Planet. Sci. Lett. 304, 93-102 (2011).

36. Ames, D. E., Franklin, J. M. \& Hannington, M. Mineralogy and geochemistry of active and inactive chimneys and massive sulphide, middle valley, northern juan de fuca ridge: an evolving hydrothermal system. Can. Mineral. 31, 997-1024 (1993).

37. Bemis, K., Lowell, R. P. \& Farough, A. Diffuse flow on and around hydrothermal vents at mid-ocean ridges. Oceanography 25, 182 (2012).

38. Seyfried, Jr W. E., Pester, N. J., Tutolo, B. M. \& Ding, K. The Lost City hydrothermal system: constraints imposed by vent fluid chemistry and reaction path models on subseafloor heat and mass transfer processes. Geochem. Cosmochim. Acta 163, 59-79 (2015).

39. Baker, E. T. Hydrothermal cooling of midocean ridge axes: do measured and modeled heat fluxes agree? Earth Planet. Sci. Lett. 263, 140-150 (2007).

40. Lowell, R. \& Rona, P. Seafloor hydrothermal systems driven by the serpentinization of peridotite. Geophys. Res. Lett. 29, 26-1-26-4 (2002). 
41. Lowell, R. P. in Diversity Of Hydrothermal Systems On Slow Spreading Ocean Ridges Vol. 188 (eds Rona, P., Dewey, C. F., Dyment, J. \& Murton, B. J.) 11-26 (American Geophysical Union, 2010).

42. Allen, D. E. \& Seyfried, Jr W. E. Serpentinization and heat generation: constraints from Lost City and Rainbow hydrothermal systems. Geochem. Cosmochim. Acta 68, 1347-1354 (2004).

43. Henstock, T. J., Woods, A. W. \& White, R. S. The accretion of oceanic crust by episodic sill intrusion. J. Geophys. Res. 98, 4143-4161 (1993).

44. Quick, J. E. \& Denlinger, R. P. Ductile deformation and the origin of layered gabbro in ophiolites. J. Geophys. Res. 98, 14015-14027 (1993).

45. McCaig, A. M., Cliff, R. A., Escartin, J., Fallick, A. E. \& MacLeod, C. J. Oceanic detachment faults focus very large volumes of black smoker fluids. Geology 35, 935-938 (2007).

46. Baker, E. T. \& German, C. R. in Mid-Ocean Ridges: Hydrothermal Interactions between the Lithosphere and Oceans, Geophysical Monograph Series Vol. 148 (eds Lin, C. R., Parson, J. \& German, L. M.) 245-266 (AGU, 2004).

47. Mottl, M. J. Explanatory notes and master chemical item. Spreadsheet for the VentDB data collections housed in the EarthChem Library. EarthChem Library. doi:10.1594/IEDA/100213 (2012).

48. Rona, P. A. \& Trivett, D. A. Discrete and diffuse heat transfer atashes vent field, Axial Volcano, Juan de Fuca Ridge. Earth Planet. Sci. Lett. 109, 57-71 (1992).

49. Converse, D. R., Holland, H. D. \& Edmond, J. M. Flow rates in the axial hot springs of the East Pacific Rise $\left(21^{\circ} \mathrm{N}\right)$ : implications for the heat budget and the formation of massive sulfide deposits. Earth Planet. Sci. Lett. 69, 159-175 (1984).

50. Ramondenc, P., Germanovich, L. N., Von Damm, K. L. \& Lowell, R. P. The first measurements of hydrothermal heat output at $950^{\prime} \mathrm{N}$, East Pacific Rise. Earth Planet. Sci. Lett. 245, 487-497 (2006).

51. Ginster, U., Mottl, M. J. \& Von Herzen, R. P. Heat flux from black smokers on the Endeavour and Cleft segments, Juan de Fuca Ridge. J. Geophys. Res. 99, 4937-4950 (1994).

52. Macdonald, K. C., Becker, K., Spiess, F. N. \& Ballard, R. D. Hydrothermal heat flux of the 'black smoker' vents on the East Pacific Rise. Earth Planet. Sci. Lett. 48, 1-7 (1980).

53. Bischoff, J. L. \& Rosenbauer, R. J. An empirical equation of state for hydrothermal seawater (3.2 percent $\mathrm{NaCl}$ ). Am. J. Sci. 285, 725-763 (1985).

54. Humphris, S. E. \& Bach, W. On the Sr isotope and REE compositions of anhydrites from the TAG seafloor hydrothermal system. Geochem. Cosmochim. Acta 69, 1511-1525 (2005).

55. Yalçin, H. \& Bozkaya, Ö. Mineralogy and geochemistry of Paleocene ultramafic- and sedimentary-hosted talc deposits in the southern part of the Sivas Basin, Turkey. Clay Clay Miner. 54, 333-350 (2006).

56. Zierenberg, R. A. \& Shanks, III W. in Geologic, Hydrothermal, and Biologic Studies at Escanaba Trough, Gorda Ridge, Offshore Northern California (eds J. Morton, R. A. Zierenberg, \& C. A. Reiss) 257-277 (U.S. G.P.O., 1994).
57. Connelly, D. P. et al. Hydrothermal Activity and Deep-Ocean Biology of the Mid-Cayman Rise. 100 (National Oceanography Centre Southampton, 2012).

58. Copley, J. T. et al. Montego Bay to Antigua, Hydrothermal Activity and Deep-Ocean Biology of the Mid-Cayman Rise (National Oceanography Centre Southampton, 2013).

59. Charlou, J. L., Donval, J. P., Fouquet, Y., Jean-Baptiste, P. \& Holm, N. Geochemistry of high $\mathrm{H} 2$ and $\mathrm{CH} 4$ vent fluids issuing from ultramafic rocks at the Rainbow hydrothermal field ( $36^{\circ} 14^{\prime} \mathrm{N}$, MAR). Chem. Geol. 191, 345-359 (2002).

\section{Acknowledgements}

The work published in this study was funded using NERC grants NE/F017758/1 and NE/I012613/1 to B.J.M. and S.R. at the National Oceanography Centre. We thank the JC44 and JC82 shipboard parties for data collection and sample acquisition. We thank R. Williams and R. Pearce for their help with the X-ray diffraction and M. Cooper, J. Hawkes and V. Chavagnac for analysing the vent fluids. We are grateful to two anonymous reviewers and $\mathrm{M}$. Palmer for comments on the manuscript.

\section{Author contributions}

M.R.S.H. conducted the petrographic, geochemical and mineral analyses, and heat flux calculations; A.P.W. made the thermodynamic modelling; R.A.M. conducted vent fluid analysis. B.J.M. and S.R. conceived and directed the research; M.R.S.H., A.P.W., B.J.M. S.R., D.P.C. and R.A.M. wrote the manuscript, and all contributed to collection of the data on cruises JC44 and JC82.

\section{Additional information}

Supplementary Information accompanies this paper at http://www.nature.com/ naturecommunications

Competing financial interests: The authors declare no competing financial interests.

Reprints and permission information is available online at http://npg.nature.com/ reprintsandpermissions/

How to cite this article: Hodgkinson, M. R. S. et al. Talc-dominated seafloor deposits reveal a new class of hydrothermal system. Nat. Commun. 6:10150 doi: $10.1038 /$ ncomms10150 (2015).

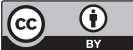

This work is licensed under a Creative Commons Attribution 4.0 International License. The images or other third party material in this article are included in the article's Creative Commons license, unless indicated otherwise in the credit line; if the material is not included under the Creative Commons license, users will need to obtain permission from the license holder to reproduce the material. To view a copy of this license, visit http://creativecommons.org/licenses/by/4.0/ 